S. Miller (U. College, London), A. Storrs (STScI, Baltimore), H. Weaver (STScI, Baltimore)

We present Hubble Space Telescope (HST) far-ultraviolet images of Jupiter over 13 July - 9 August 1994 during the period of impacts of Comet ShoemakerLevy 9 with the Wide Field Planetary Camera (WFPC2) and the Faint Object Camera (FOC). We have imaged Jupiter at far-ultraviolet (FUV) wavelengths less than 2100 Angstroms to study the response of Jupiter's upper atmosphere to the impacts, to measure upper atmospheric winds through observed motions of impact-related absorbers, and to search for auroral emissions associated with the cometary material passing through Jupiter's magnetosphere or from the impacts. We have detected extraordinary extended absorption regions at all large impact sites in the Rayleigh scattered solar continuum over 1700-2100 A. In the FUV images the impact regions were considerably darker, more extended, and more diffuse with less central contrast than at longer wavelengths, and were observed to actively darken over 2-3 hours post-impact. This indicates the presence of gases or aerosols which were more spatially extended, had a stronger absorption in the FUV than at visible wavelengths, and were likely to be at higher altitudes. Although the impact feature centers remained generally fixed in system III longitude, the initial dispersions of FUV absorbing material in the $G, L$, and $K$ features showed stronger absorptions and more pronounced north/south extensions, with initial motions of the order of $1 \mathrm{~km} / \mathrm{sec}$. We have detected transient northern auroral emissions at unusually low magnetic latitudes just after the $K$ impact, with similar but fainter emissions at either side of the $\mathrm{K}$ impact site in the southern hemisphere. The northern emissions appeared to fall near the magnetic conjugate point of the $\mathrm{K}$ impact site along $2 \mathrm{RJ}$ magnetic field lines, initially with $40 \mathrm{kR}$ brightness and decreasing with time. We have noted fainter than average global auroral activity before and during the period of collisions, which returned to a normally bright level several days after the end of the impacts, although its relationship to the event is not straightforward. Finally, we present a variable auroral emission feature along the 'normal' southern auroral oval roughly $1 / 2$ hour before the P2 impact.

\title{
HST SPECTROSCOPIC OBSERVATIONS OF JUPITER AFTER THE COLLISION OF COMET P/SHOEMAKER-LEVY 9
}

K.S. Noll, M.A. McGrath (STScI, Baltimore), L.M. Trafton (U. Texas, Austin), S.K. Atreya (U. Michigan, Ann Arbor), J.J. Caldwell (Space Astrophys. Lab., North York, Ontario), H.A. Weaver (STScI, Baltimore), R.V. 
Yelle (NASA/Ames, Moffett Field), C. Barnet (Space Astrophys. Lab., North York, Ontario), S. Edgington (U. Michigan, Ann Arbor)

The composition of Jupiter's stratosphere and upper troposphere was dramatically altered by the impacts of the fragments of comet P/ShoemakerLevy 9. UV spectra obtained with the Hubble Space Telescope have identified at least ten molecules and atoms in the vicinity of impact sites, most never before observed in Jupiter. The large mass of sulfur-containing material, more than $10^{14} \mathrm{~g}$ in $\mathrm{S}_{2}$ alone, and the relative abundances of sulfur-bearing gases, indicates that many of the sulfur-containing molecules $\mathrm{S}_{2}, \mathrm{CS}_{2}, \mathrm{CS}, \mathrm{H}_{2} \mathrm{~S}$, and $\mathrm{S}^{+}$may be derived from a sulfur-bearing parent molecule native to Jupiter. If so, this is the first observation of any sulfur compound in Jupiter and is an observational confirmation of models of cloud composition and possibly cloud coloration. The presence of jovian sulfur also constrains the depth of penetration of the fragments with the requirement that at least some must reach the expected $\mathrm{NH}_{4} \mathrm{SH}$ cloud at $\mathrm{P} \sim 1-2$ bars. For the sulfur to be derived from the impactor, a mass equivalent to a $3 \mathrm{~km}$ diameter comet or asteroid must be deposited in Jupiter's stratosphere above 100 mbar. The presence of stratospheric $\mathrm{NH}_{3}$ is also consistent with relatively deep penetration of the impactors. Neutral and ionized metals were observed in emission, including MgII, MgI, SiI, FeI, and FeII. The mass required is easily derived from a modest sized impactor. Oxygen-containing molecules were significant by their absence with the possible significant exception of $\mathrm{H}_{2} \mathrm{O}$. We derive upper limits for $\mathrm{SO}_{2}, \mathrm{SO}, \mathrm{CO}, \mathrm{SiO}$, and $\mathrm{H}_{2} \mathrm{O}$.

\section{W. M. KECK TELESCOPE OBSERVATIONS OF THE COMET P/SHOEMAKER-LEVY 9 FRAGMENT R JUPITER COLLISION}

I. de Pater, J.R. Graham, J.G. Jernigan, M.C. Liu, M.E. Brown, (U. California, Berkeley, USA)

We used the W. M. Keck telescope in Hawaii, the largest optical telescope in the world ( $10 \mathrm{~m}$ diameter) to observe the impacts of Comet P/ShoemakerLevy 9 with Jupiter. Our most surprising result was the impact of fragment $R$, which we recorded in the infrared at $2.3 \mu \mathrm{m}$ on July $21 \mathrm{UT}$. Our data, which consist of a sequence of images every $7.7 \mathrm{~s}$, show three outbursts. The first flash lasts about $40 \mathrm{~s}$, and is followed one minute after its peak by a second flash which lasts about three minutes. A third, longer lasting flare, begins brightening six minutes after the first flash, and lasts for ten minutes. At its maximum brightness the flare outshines Jupiter at this wavelength. All events 